\title{
Mathematical Modelling of Biomechanical Interactions between Backpack and Bearer during Load Carriage
}

\author{
Lei Ren, ${ }^{1}$ David Howard, ${ }^{2}$ and Richard K. Jones ${ }^{2}$ \\ ${ }^{1}$ School of Mechanical, Aerospace and Civil Engineering, University of Manchester, Manchester M60 1QD, UK \\ ${ }^{2}$ Centre for Rehabilitation and Human Performance Research, University of Salford, Salford M5 4WT, UK \\ Correspondence should be addressed to Lei Ren; lei.ren@manchester.ac.uk
}

Received 11 March 2013; Accepted 27 March 2013

Academic Editor: Chang-Hwan Im

Copyright (C) 2013 Lei Ren et al. This is an open access article distributed under the Creative Commons Attribution License, which permits unrestricted use, distribution, and reproduction in any medium, provided the original work is properly cited.

\begin{abstract}
This paper proposes a three-dimensional mathematical model of the biomechanical interactions between backpack and bearer during load carriage. The model considers both the coupled pack motions, which follow the torso, and also the longitudinal compliance and damping in the backpack suspension. The pack interaction forces and moments, acting on the bearer, are determined from kinematic relationships, equations of motion, and a dynamic pack suspension model. The parameters of the pack suspension model were identified from test data obtained using a load carriage test rig. Output from the load carriage mathematical model has been compared with measurement data during human gait and conclusions drawn with regard to the validity of the proposed approach.
\end{abstract}

\section{Introduction}

Backpacks are common devices for increasing human load carriage performance but when heavily loaded may lead to excessive joint loadings, muscle fatigue, or even injury [1-5]. Most studies of personal load carriage systems have focused on physiological and biomechanical aspects [6-10]. Biomechanical studies have concentrated on experimental gait analysis, including the effects of load carriage on electromyographic activity $[3,8,11]$, gait and posture $[6,7,10]$, and ground reactions $[6,8,9]$. Almost all of these studies consider the backpack to be part of the trunk segment, with no relative motion between the two. Little is known about the pack interaction forces and moments acting on the bearer's trunk.

During load carriage, the pack interaction forces exerted on the torso relate directly to perceived discomfort, fatigue, and the risk of injury, for example, rucksack palsy and back problems $[12,13]$. A better understanding of these interaction forces would help to improve the design of future load carriage systems. Unfortunately, in contrast to the contact pressure distribution [14-16], the interaction forces and moments between pack and torso cannot be measured directly; however mathematical modelling and simulation offer an alternative approach.

Very little has been published on the modelling of load carriage biomechanics. While examining the effects of pack load on back muscle EMG, Bobet and Norman [3] considered the pack to be part of the trunk segment for inverse dynamics purposes. Recently, Pelot et al. [17] developed a two-dimensional backpack model to investigate the forces at the shoulder and hip belts. However, their model assumes no relative movement between pack and torso and can only be used to evaluate static forces. To properly model backpack dynamics, it is necessary to include the relative motions between pack and torso, which is capable of representing the dynamic impacts on the bearer rather than static interactions only.

This paper proposes a mathematical load carriage model, which represents the three-dimensional biomechanical interactions between pack and torso. All pack motions are directly coupled to those of the bearer's trunk, with the exception of longitudinal motion up and down the bearer's back, which depends on a pack suspension model, the parameters of which were identified from test data obtained using a load carriage test rig [18]. The validity of the proposed 


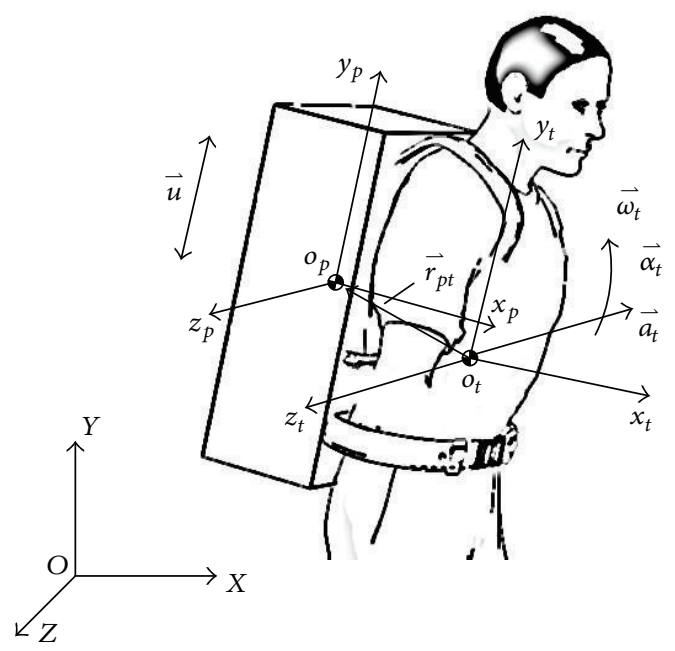

FIgURE 1: The 3D pack and trunk motions during load carriage and the three coordinate systems. OXYZ is the global (inertial) coordinate system, $o_{t} x_{t} y_{t} z_{t}$ is the trunk coordinate system, and $o_{p} x_{p} y_{p} z_{p}$ is the backpack coordinate system.

approach is investigated and conclusions drawn with regard to future developments in the modelling of load carriage biomechanics.

\section{Methods}

2.1. Pack-Trunk Kinematics. Throughout this study, the typical backpack system with two shoulder straps and a waist belt is addressed. If deformations of the backpack are neglected, the pack can be considered to be a rigid body (Figure 1). Due to the actions of the shoulder straps and hip belt, holding the pack against the bearer's back, pack rotations relative to the trunk in the sagittal and transverse planes, and translation perpendicular to the back are all constrained. For simplicity, relative pack rotation in the frontal plane and lateral pack translation are also considered to be negligible. In other words, pack motion relative to the trunk only occurs in a direction which coincides with the trunk's longitudinal axis. So, the backpack is modelled as a rigid body that slides up and down the bearer's back while all other degrees of freedom follow the motions of the trunk.

A global coordinate system $O X Y Z$, a trunk system $o_{t} x_{t} y_{t} z_{t}$, and a backpack system $o_{p} x_{p} y_{p} z_{p}$ are defined (Figure 1). The global system's $x$-axis lies in the sagittal plane and points in the direction of forward progress, the $y$-axis also lies in the sagittal plane and points upwards, and the $z$ axis lies in the frontal plane and points to the right. The trunk coordinate system $o_{t} x_{t} y_{t} z_{t}$ is attached to the bearer's trunk with its origin located at the trunk centre of mass (CoM). The $y_{t}$-axis is parallel to the trunk longitudinal axis (midpoint between $\mathrm{C} 7$ and T8 spinous process to the midpoint between xiphoid process and jugular notch) and points upwards. The $x_{t}$-axis is perpendicular to the bearer's back and points in the anterior direction. The $z_{t}$-axis is perpendicular to both $x_{t}$-axis and $y_{t}$-axis and points to the right. The local pack coordinate system $o_{p} x_{p} y_{p} z_{p}$ is attached to the backpack with its origin at the pack CoM, and its axes are parallel to those of the trunk coordinate system. In the analysis below, we have followed the common practice of not expressing vector equations in any particular coordinate frame. Where vector equations have been expanded, to derive the individual components, the resulting scalar equations are expressed in a particular coordinate frame, which is stated.

Based on the rigid body pack model proposed above, the pack's kinematic relationship with the trunk can be described by

$$
\begin{gathered}
\vec{a}_{p}=\vec{a}_{t}+\vec{a}_{p t}^{n}+\vec{a}_{p t}^{\tau}+\vec{a}_{r}+\vec{a}_{r}^{c}, \\
\vec{\omega}_{p}=\vec{\omega}_{t}, \\
\vec{\alpha}_{p}=\vec{\alpha}_{t},
\end{gathered}
$$

where $\vec{a}_{p}$ is the absolute acceleration of the pack CoM; $\vec{a}_{t}$ is the absolute acceleration of the trunk CoM; $\vec{a}_{p t}^{n}$ and $\vec{a}_{p t}^{\tau}$ are the centripetal and tangential acceleration vectors, relative to the trunk CoM, of a point fixed in the trunk coordinate frame and instantaneously coincident with the pack CoM; $\vec{a}_{r}$ is the relative pack acceleration vector along the back; and $\vec{a}_{r}^{c}$ is the Coriolis acceleration vector.

The various acceleration terms in (1a) are given by

$$
\begin{gathered}
\vec{a}_{p t}^{\tau}=\vec{\alpha}_{p} \times \vec{r}_{p t}, \\
\vec{a}_{p t}^{n}=\vec{\omega}_{t} \times\left(\vec{\omega}_{t} \times \vec{r}_{p t}\right), \\
\vec{a}_{r}=\ddot{\vec{u}}, \\
\vec{a}_{r}^{c}=2 \vec{\omega}_{p} \times \dot{\vec{u}},
\end{gathered}
$$

where $\vec{r}_{p t}=(\vec{d}+\vec{u})$ is the position vector of the pack CoM relative to the trunk CoM, $\vec{u}$ is the pack translation relative to the trunk, and $\vec{d}$ is the position of the pack CoM, relative to the trunk CoM, in the unloaded condition.

Substituting (2a), (2b), (2c), and (2d) into (1a) and considering each component in the backpack coordinate system, the absolute translational accelerations of the pack $\mathrm{CoM}$ in all three directions can be derived as

$$
\begin{aligned}
a_{p x}= & a_{t x}+\alpha_{t y} d_{z}-\alpha_{t z}\left(d_{y}+u\right)+\omega_{t x} \omega_{t y}\left(d_{y}+u\right) \\
& -\left(\omega_{t y}^{2}+\omega_{t z}^{2}\right) d_{x}+\omega_{t x} \omega_{t z} d_{z}-2 \omega_{t z} \dot{u}, \\
a_{p y}= & a_{t y}+\alpha_{t z} d_{x}-\alpha_{t x} d_{z}+\omega_{t y} \omega_{t z} d_{z} \\
& -\left(\omega_{t x}^{2}+\omega_{t z}^{2}\right)\left(d_{y}+u\right)+\omega_{t x} \omega_{t y} d_{x}+\ddot{u}, \\
a_{p z}= & a_{t z}+\alpha_{t x}\left(d_{y}+u\right)-\alpha_{t y} d_{x}+\omega_{t x} \omega_{t z} d_{x} \\
& -\left(\omega_{t x}^{2}+\omega_{t y}^{2}\right) d_{z}+\omega_{t y} \omega_{t z}\left(d_{y}+u\right)+2 \omega_{t x} \dot{u} .
\end{aligned}
$$




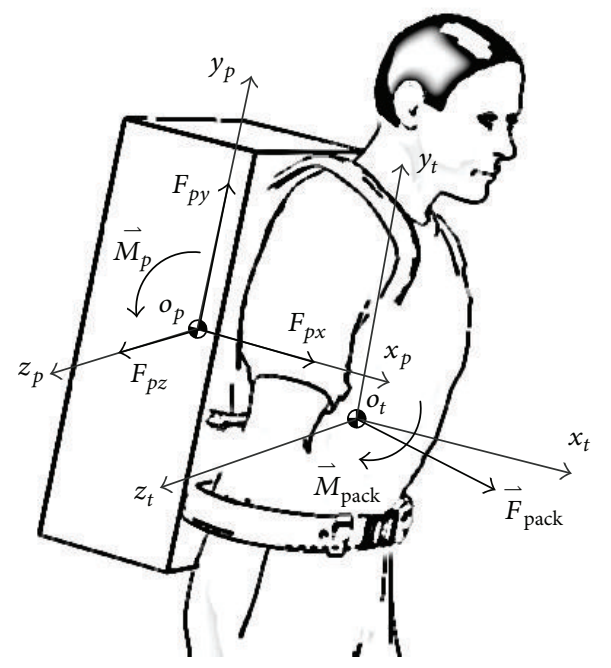

Figure 2: The human-pack interaction force $\vec{F}_{p}\left(F_{p x}, F_{p y}, F_{p z}\right)$ and moment $\vec{M}_{p}$ acting at the pack mass centre, and the associated reactions $\vec{F}_{\text {pack }}$ and $\vec{M}_{\text {pack }}$ acting at the trunk mass centre.

From (3a), (3b), and (3c), it can be seen that the backpack's absolute accelerations are not only influenced by trunk translation but also strongly coupled with trunk rotation and relative pack motion.

2.2. Backpack's Equation of Motion. If the backpack is regarded as a separate object and isolated from the torso (Figure 2), the pack interaction forces and moments can be assessed. In this case, the interaction between the pack and the body is described by the resultant force and moment $\left(\vec{F}_{p}\right.$ and $\vec{M}_{p}$ ) acting on the pack and at its CoM. This equivalent force system represents the net effects of the various forces at the pack-human interface as they act on the pack.

Applying the Newton-Euler equations leads to

$$
\begin{gathered}
m_{p} \vec{a}_{p}=\vec{F}_{p}+m_{p} \vec{g} \\
J_{p} \vec{\alpha}_{p}+\vec{\omega}_{p} \times\left(J_{p} \vec{\omega}_{p}\right)=\vec{M}_{p} .
\end{gathered}
$$

Equation (4a) can be expressed in the backpack coordinate system as

$$
\begin{gathered}
F_{p x}=m_{p} a_{p x}+m_{p} g(\sin \psi \cos \phi+\cos \psi \cos \theta \sin \phi), \\
F_{p y}=m_{p} a_{p y}+m_{p} g(\sin \psi \sin \phi+\cos \psi \cos \theta \cos \phi), \\
F_{p z}=m_{p} a_{p z}-m_{p} g \cos \psi \sin \theta,
\end{gathered}
$$

where the orientation of the backpack with respect to the global coordinate system $O X Y Z$ is defined by Euler angles $(\psi, \theta, \phi)$ in $Z X Z$ sequence.

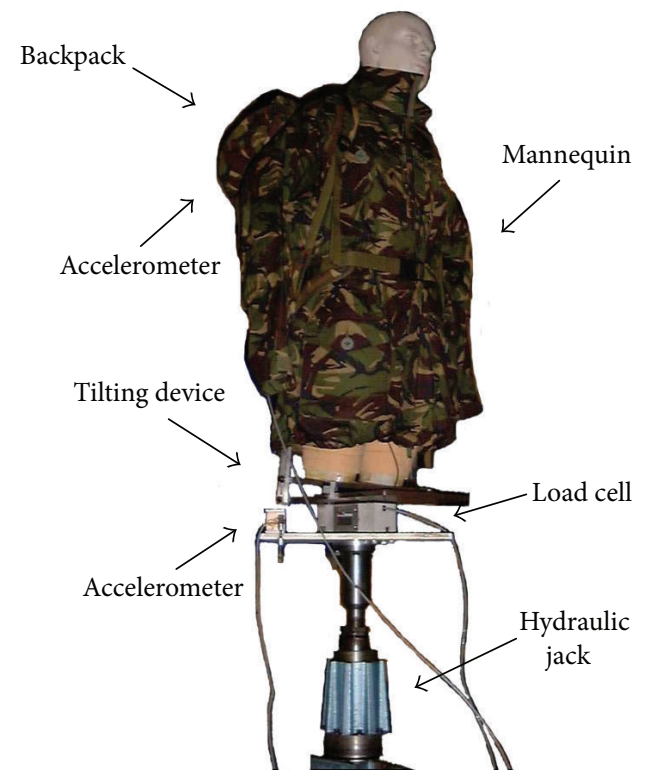

FIgURE 3: The dynamic load carriage test rig. The hydraulic ram drives the mannequin up and down at different frequencies and amplitudes. Accelerometers measure the motion of the backpack and the mannequin, and a load cell measures the dynamic force propelling the mannequin.

2.3. Backpack Suspension Model. In this study, a general nonlinear pack suspension model is employed, which relates the pack interaction force along the trunk longitudinal axis to the relative pack motion. The relationship can be written as

$$
F_{p y}=F_{p e}+F_{p d}+F_{p i}
$$

where $F_{p e}$ is the elastic component of the interaction force, $F_{p d}$ is the damping component, and $F_{p i}$ is an inertial coupling term. Cubic polynomials are used to describe these nonlinear properties [19] as follows:

$$
\begin{aligned}
& F_{p e}=a_{3} u^{3}+\operatorname{sign}(u) a_{2} u^{2}+a_{1} u, \\
& F_{p d}=b_{3} \dot{u}^{3}+\operatorname{sign}(\dot{u}) b_{2} \dot{u}^{2}+b_{1} \dot{u}, \\
& F_{p i}=c_{3} \ddot{u}^{3}+\operatorname{sign}(\ddot{u}) c_{2} \ddot{u}^{2}+c_{1} \ddot{u},
\end{aligned}
$$

where $a_{1}, a_{2}, a_{3}, b_{1}, b_{2}, b_{3}, c_{1}, c_{2}, c_{3}$ are model parameters, which are constant for a given type of pack and specified working conditions (i.e., pack load, load distribution, strap and belt tensions, etc.).

For a particular backpack, the parameters of the suspension model can be identified from dynamic test data, obtained using the hydraulically driven load carriage test rig shown in Figure 3 [19]. The mannequin was covered in a neoprenelike material to mimic the soft tissues. The hydraulic ram drives the mannequin up and down with different frequency and amplitude inputs, which allows non-linear frequency response testing over a range of frequencies and amplitudes. 


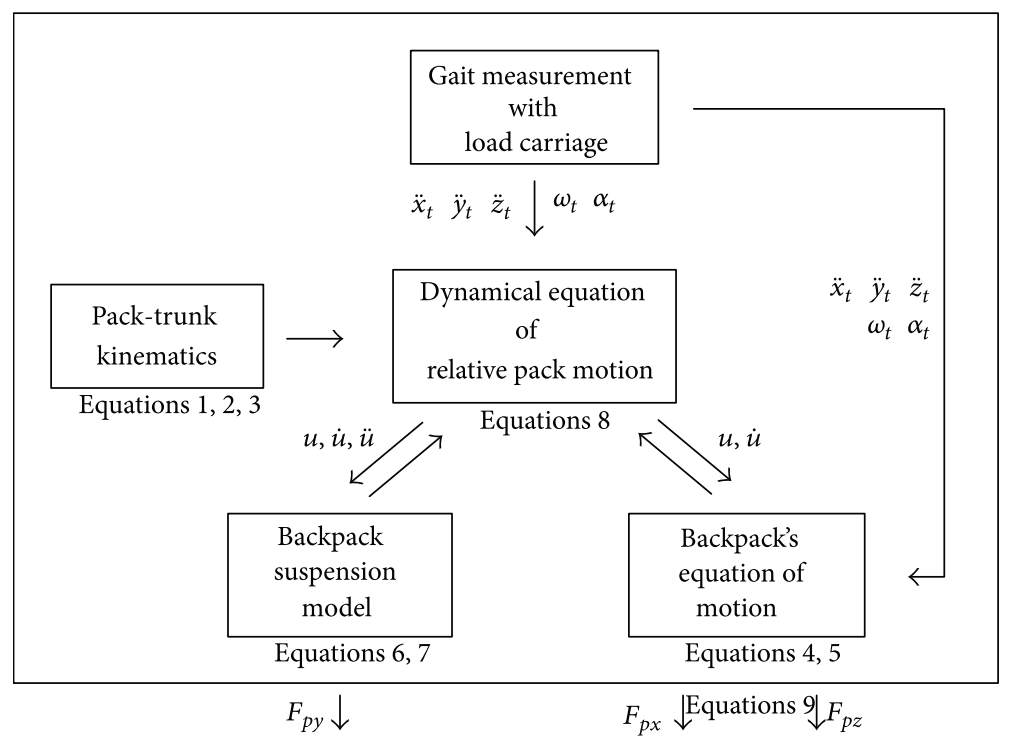

FIGURE 4: The schematic diagram for the pack-bearer interaction force calculation based on pack-trunk kinematics, backpack's equation of motion, and backpack suspension model.

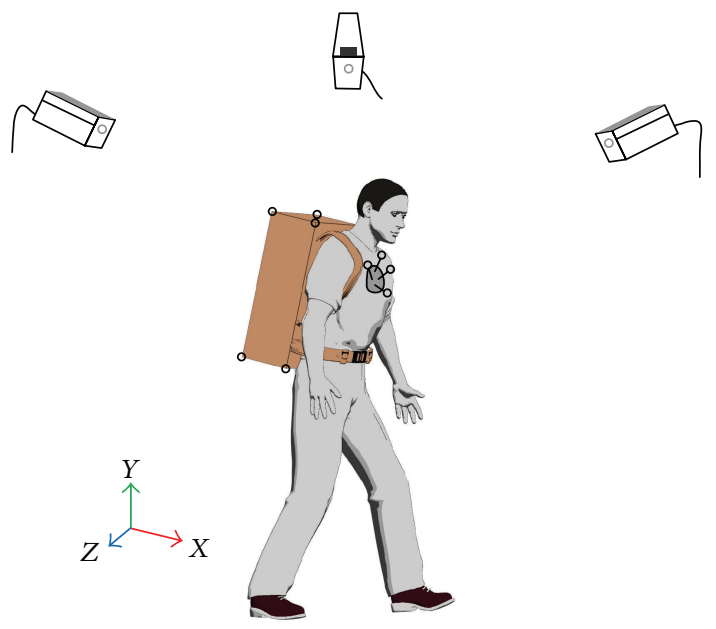

Figure 5: The 3D gait measurement setup for capturing the trunk and pack motions during load carriage. A specially designed plastic plate carrying four markers, firmly attached to the thorax, was used to capture the position and orientation of the trunk. Eight markers were attached to the corners of the pack to capture its position and orientation.

The harmonic analysis method was used to identify the model parameters [18].

2.4. Pack-Bearer Interaction Forces during Walking. Using (1a), (1b), and (1c) to (7) and measured trunk motion data, the pack interaction forces and moments can be derived as follows. Substituting the expression for acceleration $a_{p y}$ (3b) into the pack's equation of motion (5b), and using the backpack suspension model (6) and (7) to substitute for $F_{p y}$, leads to the following non-linear second-order differential equation, which describes the dynamic pack behaviour along the bearer's back:

$$
\begin{gathered}
c_{3} \ddot{u}^{3}+c_{2} \operatorname{sign}(\ddot{u}) \ddot{u}^{2}+\left(c_{1}+m_{p}\right) \ddot{u}+b_{3} \dot{u}^{3}+b_{2} \operatorname{sign}(\dot{u}) \dot{u}^{2} \\
+b_{1} \dot{u}+a_{3} u^{3}+a_{2} \operatorname{sign}(u) u^{2}+\left(a_{1}-m_{p}\left(\omega_{x}^{2}+\omega_{z}^{2}\right)\right) u \\
+m_{p}\left(a_{t y}+\alpha_{z} d_{x}-\alpha_{x} d_{z}+\omega_{y} \omega_{z} d_{z}\right. \\
-\left(\omega_{x}^{2}+\omega_{z}^{2}\right) d_{y}+\omega_{x} \omega_{y} d_{x} \\
+g(\sin \psi \sin \phi+\cos \psi \cos \theta \cos \phi))=0 .
\end{gathered}
$$

As this differential equation is of a highly non-linear form and some of the terms are time varying, a numerical solution is required (4th-order Runge-Kutta). Because the numerical integration time step is normally smaller than the gait measurement interval, cubic interpolation is used to provide trunk motion data at the necessary frequency. The initial values of the relative pack displacement and velocity were set to zero, and the numerical integration algorithm executed until a steady-state cyclic pack motion was achieved. Steady state was defined as the initial and final values of the state variables for one gait cycle being equal within an acceptable tolerance (1.0e -6 was used in this study).

Thus, given the trunk motion data $\left(a_{t y}, \psi, \theta, \phi, \omega_{x}, \omega_{y}\right.$, $\left.\omega_{z}, \alpha_{x}, \alpha_{z}\right)$, the relative pack motion can be derived $(u, \dot{u}$, and $\ddot{u})$. Then the pack force along the bearer's back $F_{p y}$ can be obtained by substituting the calculated relative motion $(u$, $\dot{u}$, and $\ddot{u}$ ) into (6) and (7).

The expressions for the normal pack force $F_{p x}$ and lateral pack force $F_{p z}$ are obtained by substituting the expressions 


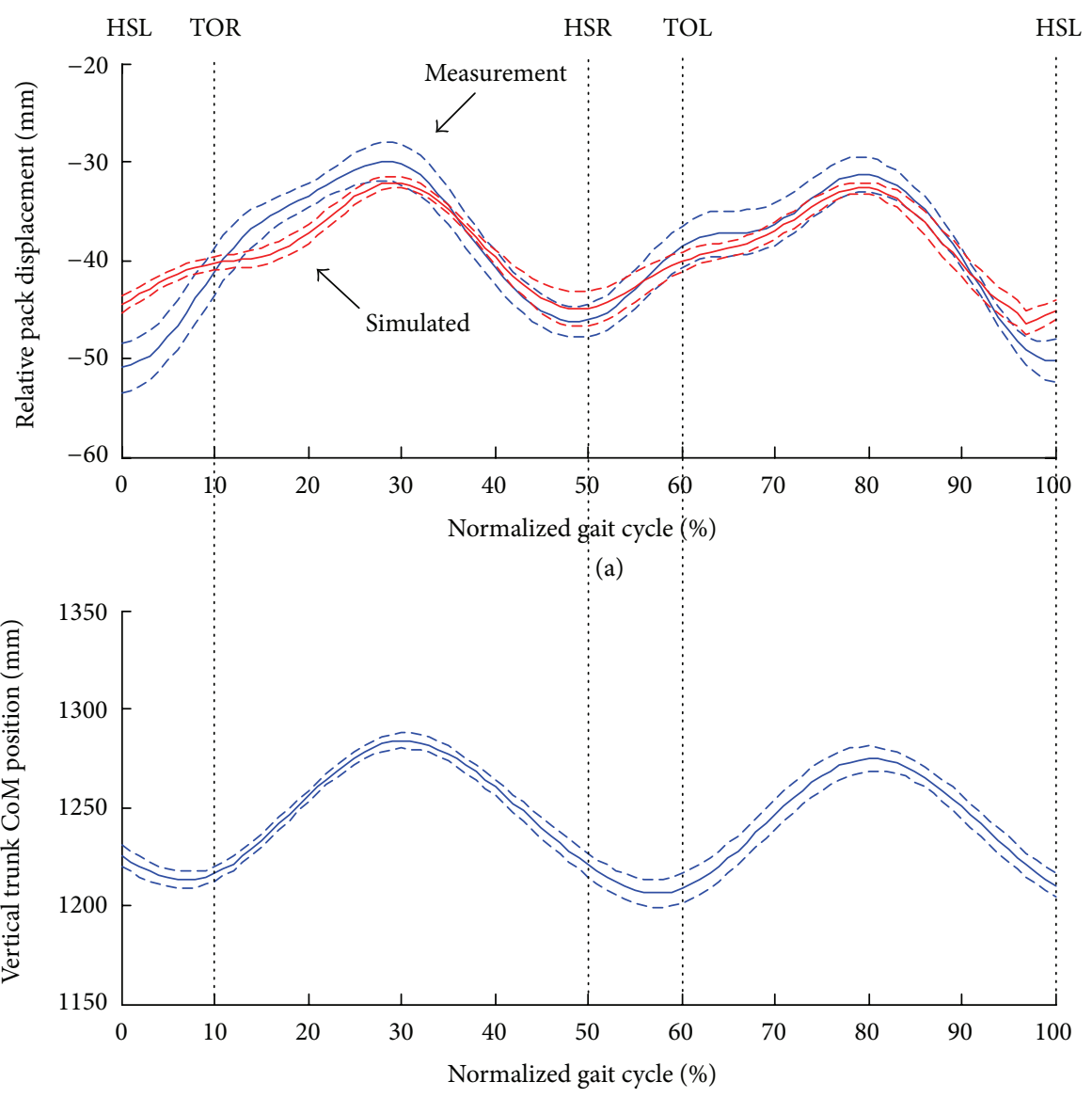

(b)

FIGURE 6: The simulated relative pack displacement along the back (mean (solid red line) \pm one standard deviation (dashed red lines), (a)), compared with measured data (mean (solid blue line) \pm one standard deviation (dashed blue lines), (a)), for six repeated trials for one subject (age: 28 yrs, weight: $71.8 \mathrm{~kg}$, height: $178 \mathrm{~cm}$ ), and measured vertical trunk CoM displacement (mean (solid blue line) \pm one standard deviation (dashed blue lines), (b)). The average walking speed was $1.63 \pm 0.03 \mathrm{~m} / \mathrm{s}$, and the pack load was $11.5 \mathrm{~kg}$. The stance phase for the left limb is from 0 to $60 \%$, and the swing phase is from $60 \%$ to $100 \%$. The double support phase is from 0 to $10 \%$ and from 50 to $60 \%$.

for accelerations $a_{p x}$ and $a_{p z}((3 \mathrm{a})$ and $(3 \mathrm{c}))$ into the pack's equations of motion $((5 \mathrm{a})$ and $(5 \mathrm{c}))$, which leads to

$$
\begin{aligned}
& F_{p x}=m_{p}\left(a_{t x}\right.+\alpha_{y} d_{z}-\alpha_{z}\left(d_{y}+u\right)+\omega_{x} \omega_{y}\left(d_{y}+u\right) \\
&-\left(\omega_{y}^{2}+\omega_{z}^{2}\right) d_{x}+\omega_{x} \omega_{z} d_{z}-2 \omega_{z} \dot{u} \\
&+g(\sin \psi \cos \phi+\cos \psi \cos \theta \sin \phi)), \\
& F_{p z}=m_{p}\left(a_{t z}+\alpha_{x}\left(d_{y}+u\right)-\alpha_{y} d_{x}+\omega_{x} \omega_{z} d_{x}\right. \\
&-\left(\omega_{x}^{2}+\omega_{y}^{2}\right) d_{z}+\omega_{y} \omega_{z}\left(d_{y}+u\right) \\
&\left.+2 \omega_{x} \dot{u}-g \cos \psi \sin \theta\right) .
\end{aligned}
$$

Thus, using (6), (7), and (9), the three components of the pack force, acting on the pack CoM, can be determined from the trunk motion data and the calculated relative pack motion. The resultant pack moment, acting about the pack
CoM, can be calculated directly from the trunk rotations, given that the pack rotates with the trunk (see (1b), (1c), and (4b)).

The pack interaction force and moment can also be represented by an equivalent force system $\left(\vec{F}_{\text {pack }}, \vec{M}_{\text {pack }}\right)$ acting on the trunk CoM (Figure 2). According to Newton's third law, the interaction force and moment acting at the trunk CoM can be expressed as

$$
\begin{aligned}
& \vec{M}_{\text {pack }}=\vec{F}_{p} \times \vec{r}_{t p}-\vec{M}_{p}, \\
& \vec{F}_{\text {pack }}=-\vec{F}_{p} .
\end{aligned}
$$

The pack force and moment can be used to assess the mechanical loads imposed on the human body during load carriage. Moreover, when input into a three-dimensional human gait model, joint loads and mechanical energy expenditure can also be evaluated.

The proposed dynamic load carriage model has been implemented in the MATLAB programming environment (see Figure 4 for a schematic diagram). Measured 3D trunk 

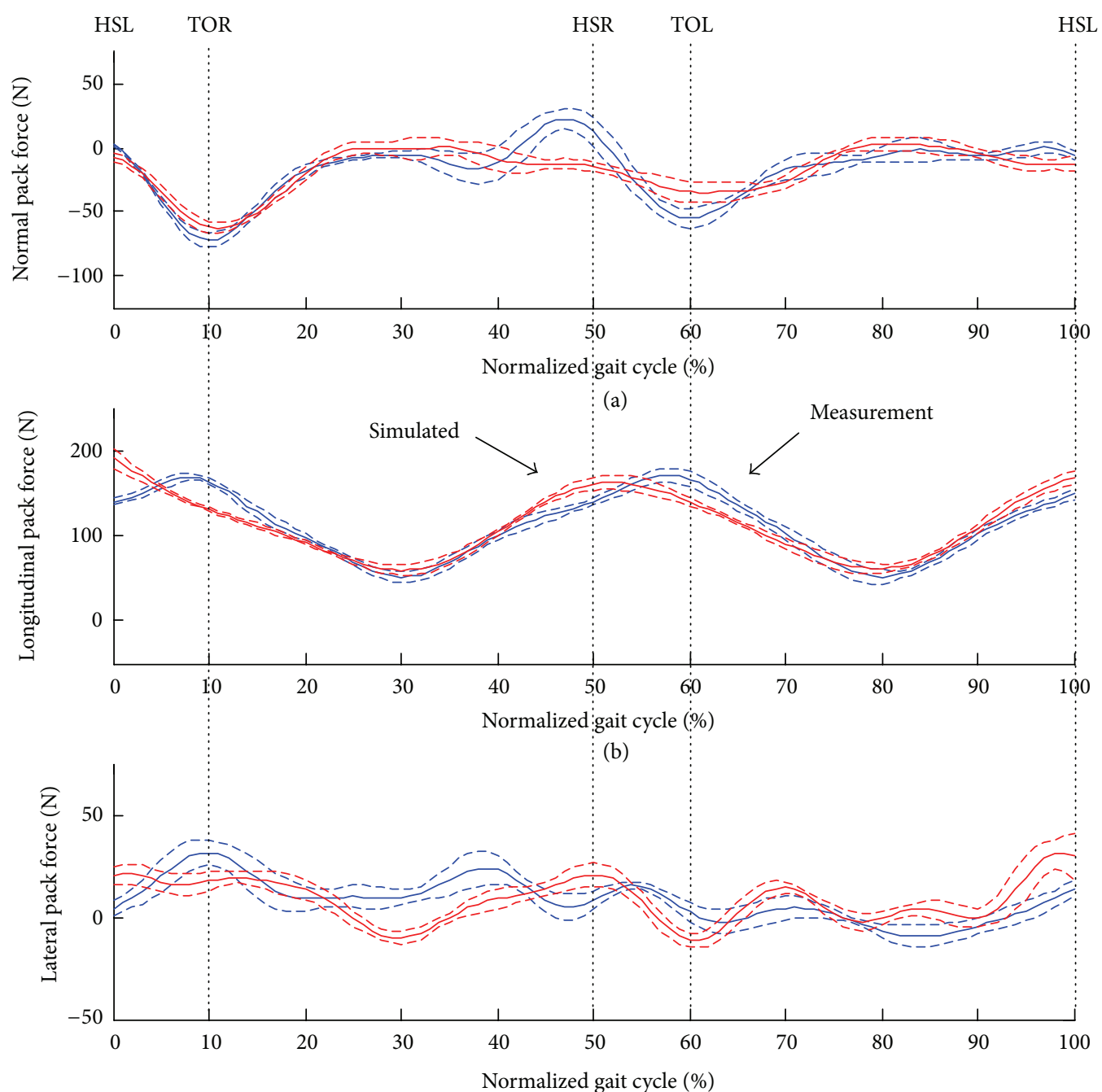

(c)

FIGURE 7: The simulated pack interaction forces in the pack coordinate system mean ((solid red line)) \pm one standard deviation (dashed red lines): (a) longitudinal pack force, (b) normal pack force (c) and lateral pack force, compared with measured data (mean (solid blue line) \pm one standard deviation (dashed blue lines)), for six repeated trials for one subject (age: $28 \mathrm{yrs}$, weight: $71.8 \mathrm{~kg}$, height: $178 \mathrm{~cm}$ ). The average walking speed was $1.63 \pm 0.03 \mathrm{~m} / \mathrm{s}$, and the pack load was $11.5 \mathrm{~kg}$. The stance phase for the left limb is from 0 to $60 \%$, and the swing phase is from $60 \%$ to $100 \%$. The double support phase is from 0 to $10 \%$ and from 50 to $60 \%$.

rotations and translations over a complete gait cycle, with a loaded backpack, were used as input data.

2.5. Experimental Model Assessment. To assess the validity of the proposed modelling approach, three-dimensional gait measurement was conducted to capture trunk and pack motions whilst carrying a backpack. Two healthy male subjects (age: $30 \pm 2$ yrs, weight: $75 \pm 3.2 \mathrm{~kg}$ ) were selected from a population of postgraduate students. Prior to participation, the subjects provided informed consent in accordance with the policies of Salford University's Ethical Advisory Committee. The subjects walked in trainers along a walkway, inside a gait laboratory, while motion data was collected at $100 \mathrm{~Hz}$ using a 6-camera Qualisys motion analysis system (Qualisys $\mathrm{AB}$, Savedalen, Sweden). The subjects walked at two different speeds, normal and fast, with two backpack loads, $11.5 \mathrm{~kg}$ and $23.0 \mathrm{~kg}$ (see Figure 5). Each experimental condition was measured six times to ensure that a repeatable data set for a complete walking cycle was obtained.

To capture trunk motions, a specially designed plastic plate, carrying four reflective markers, was firmly attached to the thorax [20]. Four anatomical landmarks (C7 and T8 spinous processes, xiphoid process, and jugular notch) were used to define the trunk coordinate system. Before the walking trials, a static calibration procedure was used to locate these anatomical landmarks based on the CAST methodology (calibrated anatomical system technique) [21]. The position of the trunk CoM was estimated from the anatomical landmarks using anthropometric data from the literature [22].

To minimize the deformation of the backpack, the test pack contained a specially designed plastic foam block with 


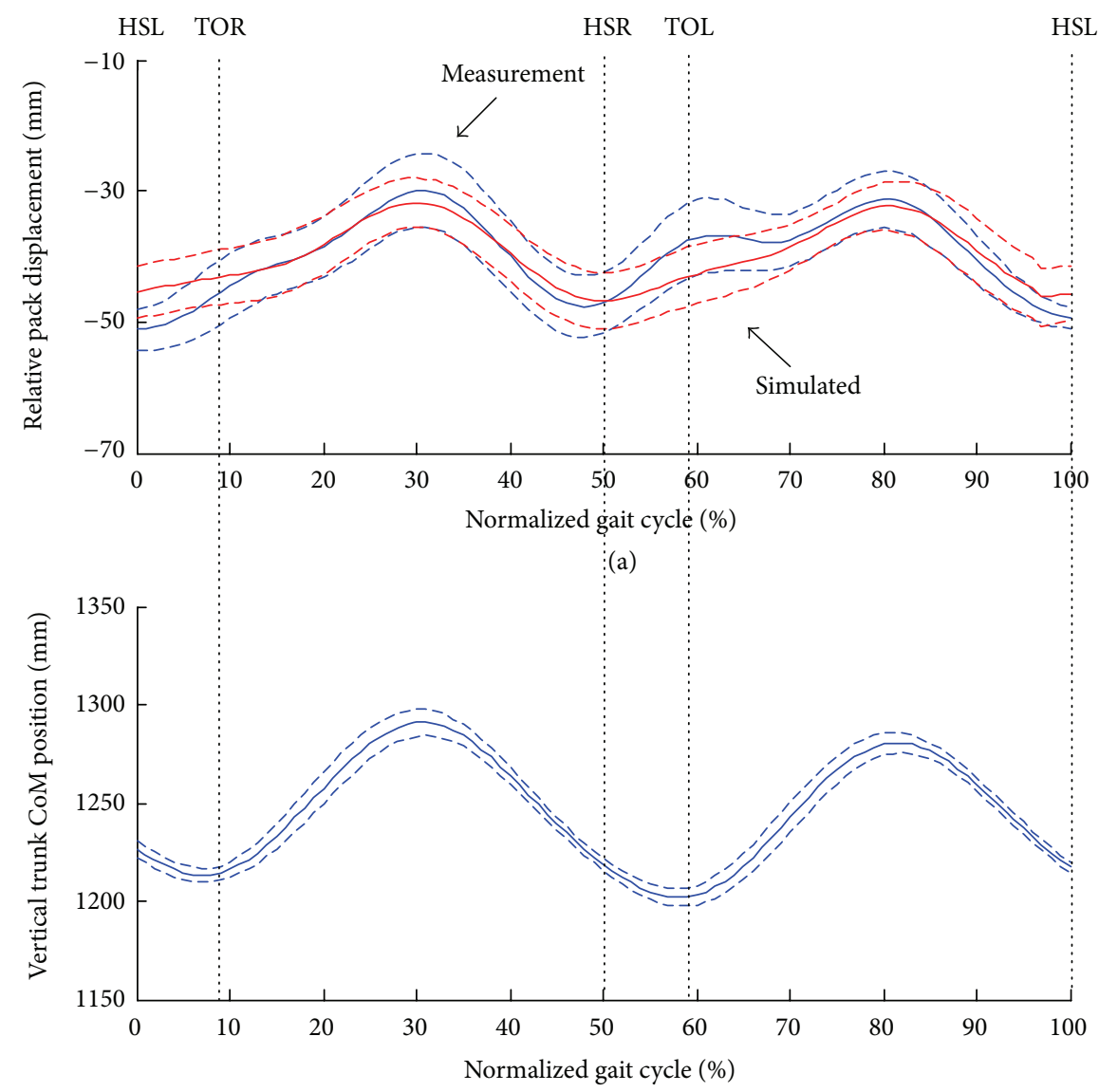

(b)

FIGURE 8: The simulated relative pack displacement along the back (mean (solid red line) \pm one standard deviation (dashed red lines), (a)), compared with measured data (mean (solid blue line) \pm one standard deviation (dashed blue lines), (a)), for six repeated trials for one subject (age: 28 yrs, weight: $71.8 \mathrm{~kg}$, height: $178 \mathrm{~cm}$ ), and measured vertical trunk CoM displacement (mean (solid blue line) \pm one standard deviation (dashed blue lines), (b)). The average walking speed was $1.93 \pm 0.03 \mathrm{~m} / \mathrm{s}$, and the pack load was $11.5 \mathrm{~kg}$. The stance phase for the left limb is from 0 to $59 \%$, and the swing phase is from $59 \%$ to $100 \%$. The double support phase is from 0 to $9 \%$ and from 50 to $59 \%$.

cylindrical cavities to allow metal bars to be firmly inserted. By putting different metal bars in different cavities, different pack loads and load distributions were possible. During walking trials, eight reflective markers were attached to the corners of the backpack, and the geometric centre of the pack was assumed to be equidistant from these markers. The pack's CoM position and inertia matrix were then calculated based on this geometric centre and the load distribution within the pack.

The raw 3D marker data were processed using a customwritten software package, SMAS (Salford Motion Analysis Software), which has been developed for 3D kinematic and kinetic analysis of general biomechanical multibody systems $[20,23]$. The data were filtered using a low pass 4 th-order Butterworth digital filter with a cut-off frequency of $15 \mathrm{~Hz}$. The orientations of the backpack and trunk segments were derived using an optimal estimation algorithm [24]. The pack and trunk angular velocities, angular accelerations, and CoM linear accelerations were calculated using a finite difference method.
Based on these measurement data, the interaction force and moment acting at the pack CoM were estimated as follows:

$$
\begin{aligned}
& \vec{F}_{p m}=m_{p}\left(\vec{a}_{p m}-\vec{g}\right), \\
& \vec{M}_{p m}=J_{p} \vec{\alpha}_{p m}+\vec{\omega}_{p m} \times\left(J_{p} \vec{\omega}_{p m}\right) .
\end{aligned}
$$

These data for the whole gait cycle were then used to validate the 3D load carriage model.

\section{Results}

Experimental and simulated data for one subject walking at two different speeds, normal $(1.6 \mathrm{~m} / \mathrm{s})$ and fast $(1.9 \mathrm{~m} / \mathrm{s})$, with two backpack loads, $11.5 \mathrm{~kg}$ and $23.0 \mathrm{~kg}$, are presented in Figures 6 to 11. Results are shown for repeated experimental trials and the corresponding simulations.

Figures 6 and 7 show the vertical trunk CoM motion, the simulated relative pack displacement, and the pack 

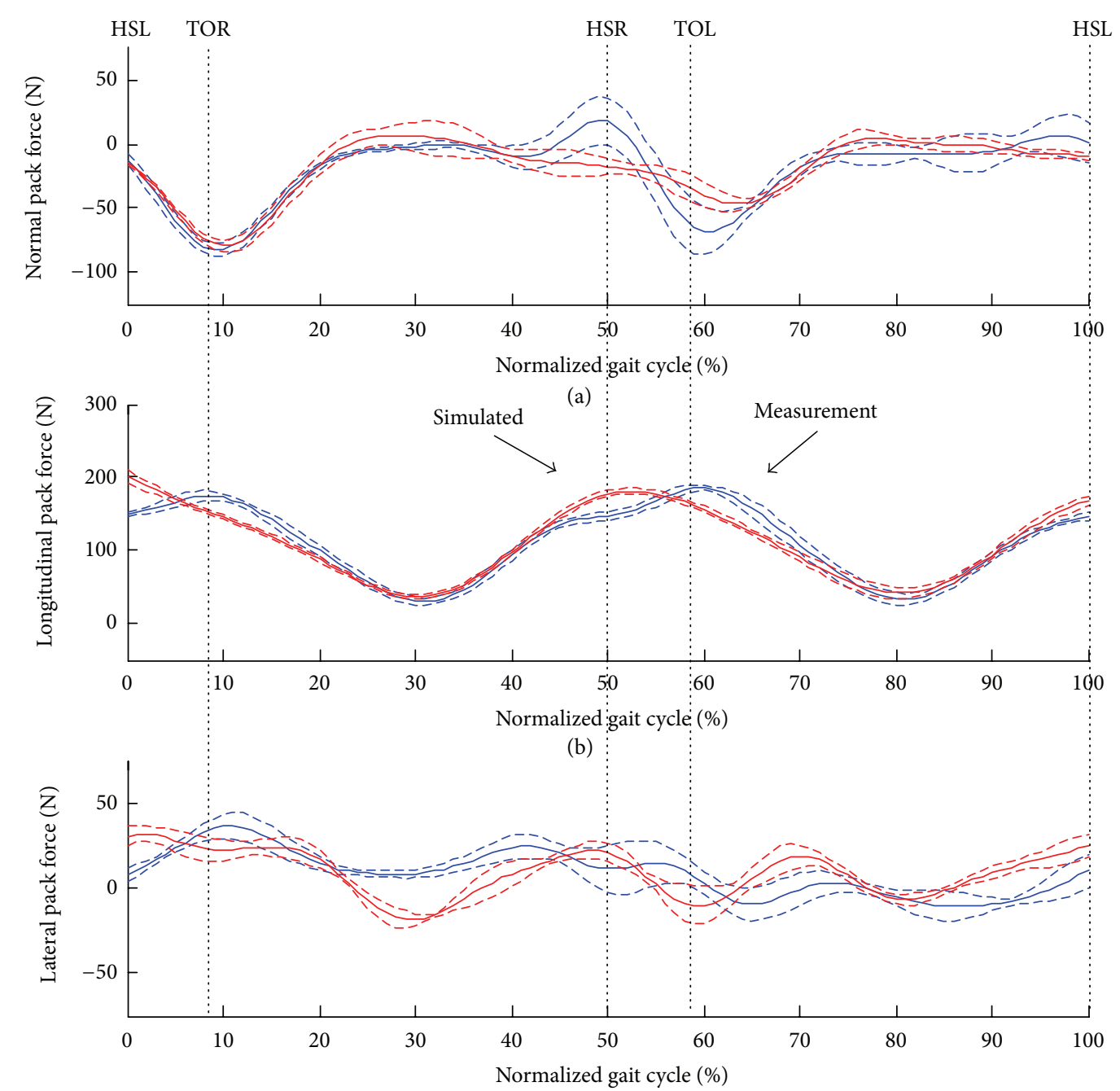

(c)

FIGURE 9: The simulated pack interaction forces in the pack coordinate system (mean (solid red line) \pm one standard deviation (dashed red lines)): (a) longitudinal pack force, (b) normal pack force (c), and lateral pack force, compared with measured data (mean (solid blue line) \pm one standard deviation (dashed blue lines)), for six repeated trials for one subject (age: 28 yrs, weight: $71.8 \mathrm{~kg}$, height: $178 \mathrm{~cm}$ ). The average walking speed was $1.93 \pm 0.03 \mathrm{~m} / \mathrm{s}$, and the pack load was $11.5 \mathrm{~kg}$. The stance phase for the left limb is from 0 to $59 \%$, and the swing phase is from $59 \%$ to $100 \%$. The double support phase is from 0 to $9 \%$ and from 50 to $59 \%$.

interaction forces, over a complete gait cycle, for normal walking with an $11.5 \mathrm{~kg}$ load. Figures 8 and 9 show the same data for fast walking and an $11.5 \mathrm{~kg}$ load. Figures 10 and 11 show the same data for normal walking with a $23 \mathrm{~kg}$ load.

\section{Discussion}

A 3D load carriage model has been proposed, which simulates the dynamic interactions between pack and bearer. This considers pack motions that are directly coupled to the bearer's trunk movement and also the longitudinal compliance and damping in the backpack suspension. The 3D pack interaction forces and moments are determined from kinematic relationships, equations of motion, and a dynamic backpack suspension model. The parameters of the pack suspension model were identified from test data collected using a load carriage test rig $[18,19]$. Output from the load carriage simulation model was compared with measurement data obtained from a motion analysis system at two different pack loads, and during both normal and fast walking.

Referring to Figures 6(a), 8(a), and 10(a), the simulated relative pack displacements (longitudinally along the bearer's back) show reasonably good agreement with the measured data at both pack loads, and at both walking speeds. They reproduce a sinusoidal fluctuation about $20 \mathrm{~mm}$ with a very similar pattern to vertical displacement of trunk CoM (Figures $6(\mathrm{~b}), 8(\mathrm{~b})$, and $10(\mathrm{~b})$ ), which is consistent with previous load carriage measurements [10]. However, the results appear to contain an additional higher-frequency harmonic that is not present in the measurement data, especially noticeable at the normal walking speed.

Referring to Figures 7(b), 9(b), and 11(b), the simulated longitudinal pack interaction forces also show reasonably good agreement with the measured data in terms of 


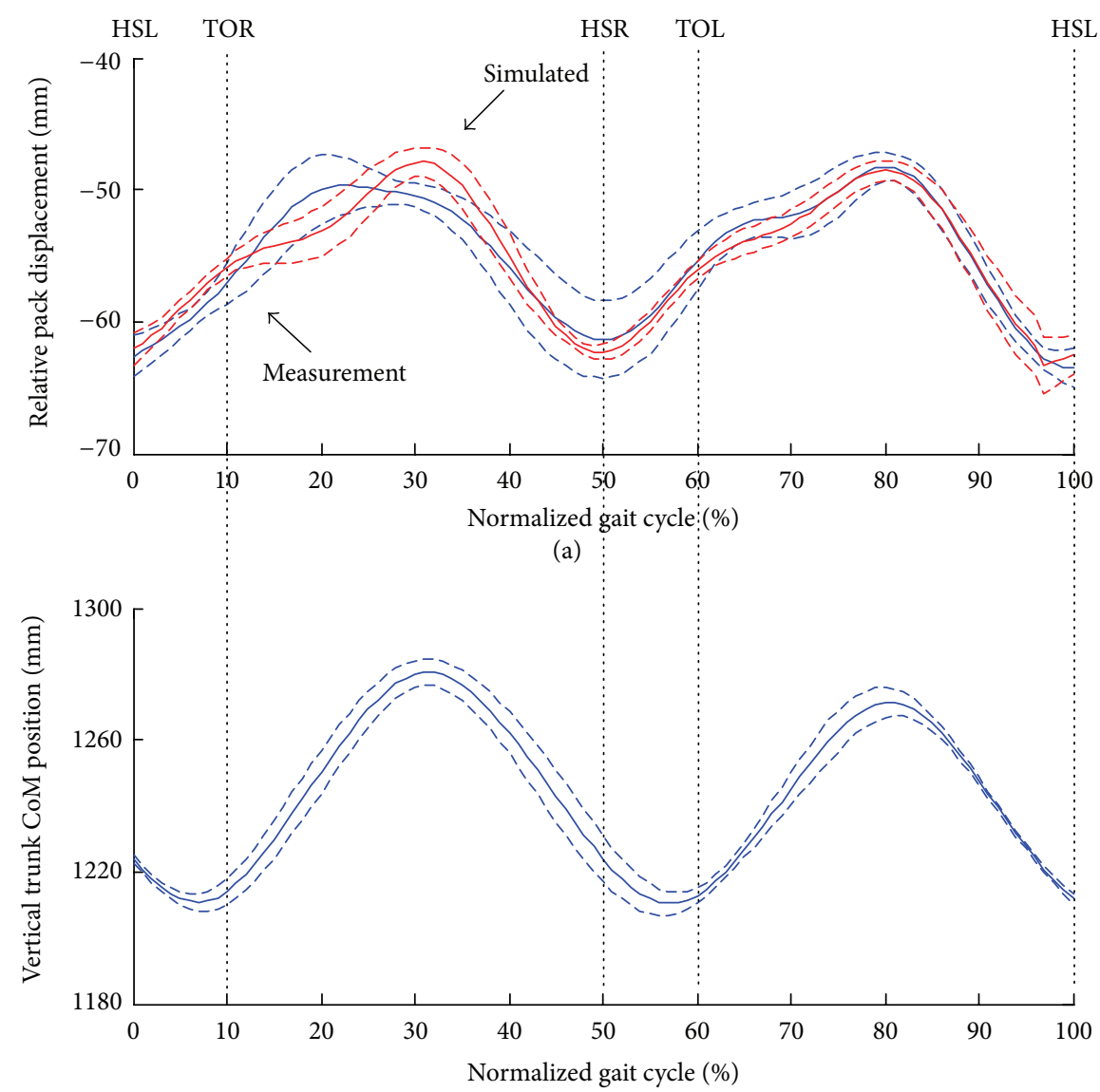

(b)

Figure 10: The simulated relative pack displacement along the back (mean (solid red line) \pm one standard deviation (dashed red lines), (a)), compared with measured data (mean (solid blue line) \pm one standard deviation (dashed blue lines), (a)), for six repeated trials for one subject (age: 28 yrs, weight: $71.8 \mathrm{~kg}$, height: $178 \mathrm{~cm}$ ), and measured vertical trunk CoM displacement (mean (solid blue line) \pm one standard deviation (dashed blue lines), (b)). The average walking speed was $1.62 \pm 0.04 \mathrm{~m} / \mathrm{s}$, and the pack load was $23.0 \mathrm{~kg}$. The stance phase for the left limb is from 0 to $60.3 \%$, and the swing phase is from $60.3 \%$ to $100 \%$. The double support phase is from 0 to $10.3 \%$ and from 50 to $60.3 \%$.

amplitudes and general trends. The largest pack forces occur during double stance because of the high accelerations at heel strike and push-off. As one would expect, the pack forces reach their minima in midstance when the vertical accelerations are negative. Both peak and mean values of the longitudinal pack forces increase significantly with increased pack load, which is consistent with the results of previous experimental load carriage studies [10]. However, the simulated longitudinal pack force also exhibits an additional harmonic which is not present in the measured data.

It is likely that the additional harmonic seen in the longitudinal results is due to the limitations of the load carriage test-rig $[18,19]$. Despite the fact that the mannequin was covered in a neoprene-like material to mimic the soft tissues, it is unlikely that there was sufficient compliance; resulting in a suspension model with a high natural frequency. Furthermore, neither the mannequin nor the simulation model can alter torso posture in response to changes in pack interaction forces. In practise, the bearer may dynamically adjust their posture to damp the pack motion and thereby decrease high-frequency fluctuations in pack force.
Furthermore, the test rig only generates vertical displacement of the mannequin, rather than the true 3D trunk motion seen during walking. Hence, the pack suspension model is limited to one degree of freedom and the suspension model parameters do not properly account for coupling with other motions.

Although the pack model neglects all relative pack motions except for longitudinal translation up and down the back, the simulated normal pack forces (Figures 7(a), $9(\mathrm{a})$, and 11(a)) were in reasonable agreement with the experimental data with the exception of unexplained discrepancies around $40 \%$ to $60 \%$ of the gait cycle. In percentage terms, there are major discrepancies between the model predictions and the measurement data in the lateral pack forces (Figures 7(c), 9(c), and 11(c)). However, these forces are of small amplitude and the experimental data exhibit significant variability.

In conclusion, whilst promising results have been achieved, the proposed methodology still needs some improvements especially the technique to identify pack suspension parameters $[18,19]$. Although the load carriage 


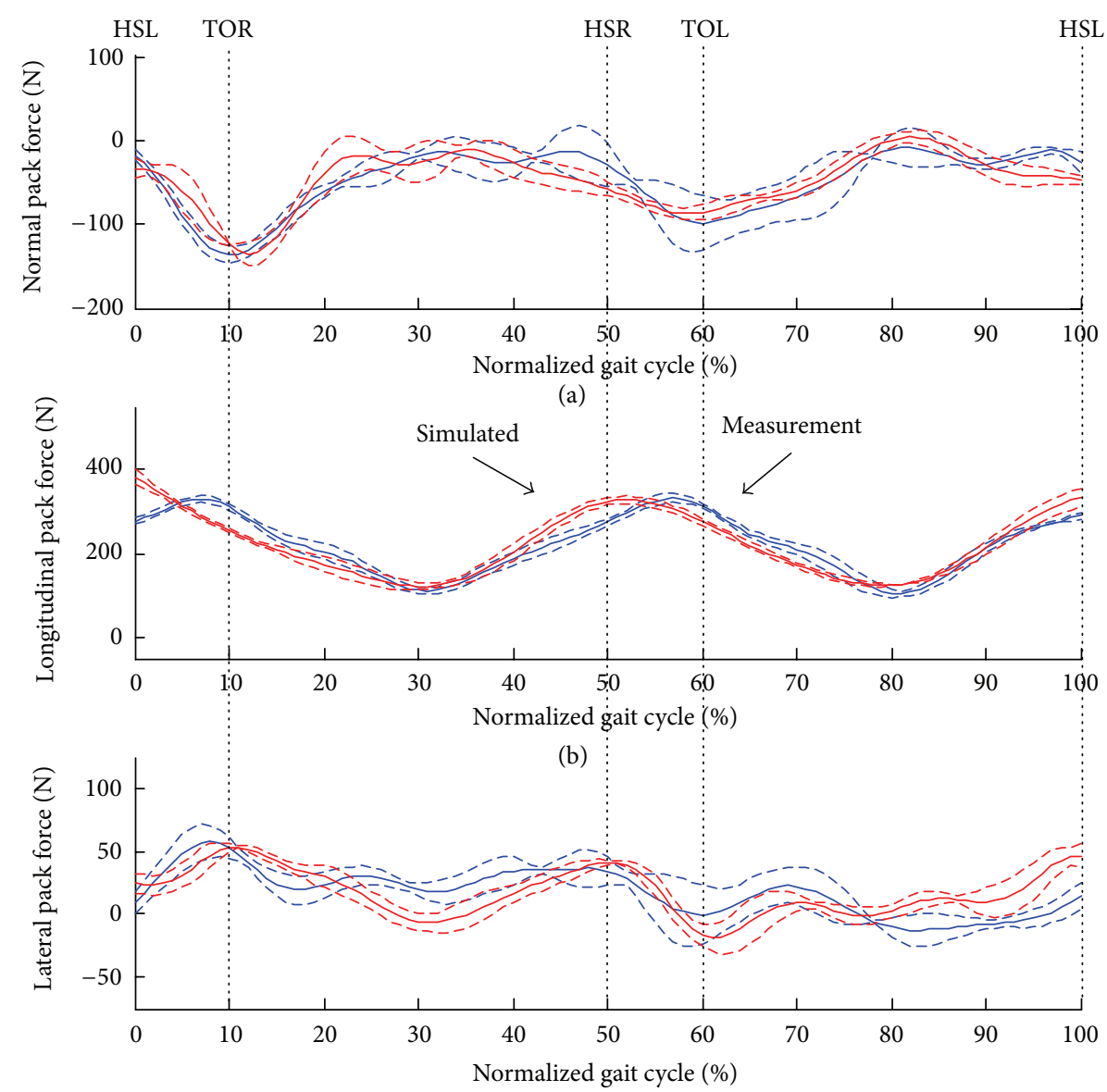

(c)

FIGURE 11: The simulated pack interaction forces in the pack coordinate system (mean (solid red line) \pm one standard deviation (dashed red lines)): (a) longitudinal pack force, (b) normal pack force (c), and lateral pack force, compared with measured data (mean (solid blue line) \pm one standard deviation (dashed blue lines)), for six repeated trials for one subject (age: 28 yrs, weight: $71.8 \mathrm{~kg}$, height: $178 \mathrm{~cm}$ ). The average walking speed was $1.62 \pm 0.04 \mathrm{~m} / \mathrm{s}$, and the pack load was $23.0 \mathrm{~kg}$. The stance phase for the left limb is from 0 to $60.3 \%$, and the swing phase is from $60.3 \%$ to $100 \%$. The double support phase is from 0 to $10.3 \%$ and from 50 to $60.3 \%$.

test-rig has the advantage that full non-linear frequency response testing can be conducted, it does not adequately represent the effective compliance of the torso. Therefore, we suggest that future work should focus on techniques for identifying backpack suspension models by combining both load carriage test-rig data and in vivo data captured in the gait laboratory. This would have the advantage that the small but significant rotations (in the plane of the back) and lateral translations of the pack, relative to the torso, could be taken into account. Including these relative motions, in addition to the longitudinal translation, may further improve the accuracy of the predicted pack forces.

\section{Nomenclature}

$\vec{a}_{p}$ : Linear acceleration vector of the pack CoM with respect to the global frame

$\vec{a}_{t}$ : Linear acceleration vector of the trunk CoM w.r.t. the global frame

$\vec{a}_{p t}^{n}$ : Centripetal acceleration vector of the pack CoM w.r.t. the trunk CoM $\vec{a}_{p t}^{\tau}$ : Tangential acceleration vector of the pack CoM w.r.t. the trunk CoM

$\vec{a}_{r}$ : Relative pack acceleration vector along the back

$\vec{a}_{r}^{c}$ : Coriolis acceleration vector induced by relative pack motion

$\vec{\omega}_{p}$ : Angular velocity of the backpack

$\vec{\alpha}_{p}$ : Angular acceleration of the backpack

$\vec{\omega}_{t}$ : Angular velocity of the trunk

$\vec{\alpha}_{t}$ : Angular acceleration of the trunk

$\vec{u}$ : Relative pack translation w.r.t. the trunk

$\vec{d}$ : Position vector of the pack CoM w.r.t. trunk CoM in unloaded condition

$\vec{r}_{p t}$ : Position vector of the pack CoM relative to the trunk COM with load

$\dot{\vec{u}}$ : Relative velocity vector of the pack w.r.t. the trunk

$\ddot{\vec{u}}$ : Relative acceleration vector of pack w.r.t. the trunk 


\begin{tabular}{|c|c|}
\hline$a_{p x}, a_{p y}, a_{p z}$ & $\begin{array}{l}\text { Linear accelerations of pack CoM in pack } \\
\text { coordinate system }\end{array}$ \\
\hline$\alpha_{p x}, \alpha_{p y}, \alpha_{p z}:$ & $\begin{array}{l}\text { Angular accelerations of pack in pack } \\
\text { coordinate system }\end{array}$ \\
\hline$a_{t x}, a_{t y}, a_{t z}$ & $\begin{array}{l}\text { Linear accelerations of trunk CoM in pack } \\
\text { coordinate system }\end{array}$ \\
\hline$\omega_{t x}, \omega_{t y}, \omega_{t z}$ & $\begin{array}{l}\text { Angular velocities of trunk in pack } \\
\text { coordinate system }\end{array}$ \\
\hline$\alpha_{t x}, \alpha_{t y}, \alpha_{t z}$ & $\begin{array}{l}\text { Angular accelerations of trunk in pack } \\
\text { coordinate system }\end{array}$ \\
\hline$d_{x}, d_{y}, d_{z}:$ & $\begin{array}{l}\text { relative positions of pack CoM w.r.t. trunk } \\
\text { CoM in pack coordinate system }\end{array}$ \\
\hline$\vec{F}_{p}:$ & $\begin{array}{l}\text { Resultant pack force vector acting at the } \\
\text { pack CoM }\end{array}$ \\
\hline$F_{p x}, F_{p y}, F_{p z}:$ & $\begin{array}{l}\text { Pack force components in pack coordinate } \\
\text { system }\end{array}$ \\
\hline$\vec{M}_{p}:$ & $\begin{array}{l}\text { Resultant pack moment vector acting at the } \\
\text { pack CoM }\end{array}$ \\
\hline$m_{p}:$ & Mass of the backpack \\
\hline$\vec{g}:$ & Gravitational acceleration vector \\
\hline$J_{p}:$ & $\begin{array}{l}\text { Inertial matrix of the backpack about the } \\
\text { pack CoM }\end{array}$ \\
\hline$\psi, \theta, \phi:$ & $\begin{array}{l}\text { Backpack Euler angles w.r.t. the global } \\
\text { coordinate system in } Z X Z \text { sequence }\end{array}$ \\
\hline$F_{p e}:$ & $\begin{array}{l}\text { Elastic component of the longitudinal pack } \\
\text { force }\end{array}$ \\
\hline$F_{p d}:$ & $\begin{array}{l}\text { Damping component of the longitudinal } \\
\text { pack force }\end{array}$ \\
\hline$F_{p i}:$ & $\begin{array}{l}\text { Inertial coupling component of the } \\
\text { longitudinal pack force }\end{array}$ \\
\hline$a_{1}, a_{2}, a_{3}:$ & $\begin{array}{l}\text { Elastic parameters of the pack suspension } \\
\text { model }\end{array}$ \\
\hline$b_{1}, b_{2}, b_{3}:$ & $\begin{array}{l}\text { Damping parameters of the pack suspension } \\
\text { model }\end{array}$ \\
\hline$c_{1}, c_{2}, c_{3}$ & $\begin{array}{l}\text { Inertial parameters of the pack suspension } \\
\text { model }\end{array}$ \\
\hline$\vec{F}_{\text {pack }}:$ & $\begin{array}{l}\text { Resultant pack force vector acting at the } \\
\text { trunk CoM }\end{array}$ \\
\hline$\vec{M}_{\text {pack }}:$ & $\begin{array}{l}\text { Resultant pack moment vector acting about } \\
\text { the trunk CoM }\end{array}$ \\
\hline$\vec{F}_{p m}:$ & $\begin{array}{l}\text { Estimated pack force from the measurement } \\
\text { data }\end{array}$ \\
\hline$\vec{M}_{p m}:$ & $\begin{array}{l}\text { Estimated pack moment from the } \\
\text { measurement data }\end{array}$ \\
\hline$\vec{a}_{p m}:$ & $\begin{array}{l}\text { Estimated linear acceleration of the pack } \\
\text { CoM from the measurement data }\end{array}$ \\
\hline$\vec{\omega}_{p m}:$ & $\begin{array}{l}\text { Estimated angular velocity of the pack from } \\
\text { the measurement data }\end{array}$ \\
\hline & $\begin{array}{l}\text { Estimated angular acceleration of the pack } \\
\text { from the measurement data. }\end{array}$ \\
\hline
\end{tabular}

\section{Conflict of Interests}

There is no conflict of interests.

\section{Acknowledgments}

This work has been supported by the UK Ministry of Defence and also the UK EPSRC from Grant no. EP/I033602/1. The assistance and support of Will Tutton (UK MoD) has been particularly valuable.

\section{References}

[1] W. S. Myles and P. L. Saunders, "The physiological cost of carrying light and heavy loads," European Journal of Applied Physiology and Occupational Physiology, vol. 42, no. 2, pp. 125131, 1979.

[2] B. H. Jones, "Overuse injuries of the lower extremities associated with marching, jogging, and running: a review," Military Medicine, vol. 148, no. 10, pp. 783-787, 1983.

[3] J. Bobet and R. W. Norman, "Effects of load placement on back muscle activity in load carriage," European Journal of Applied Physiology and Occupational Physiology, vol. 53, no. 1, pp. 71-75, 1984.

[4] J. Knapik, E. Harman, and K. Reynolds, "Load carriage using packs: a review of physiological, biomechanical and medical aspects," Applied Ergonomics, vol. 27, no. 3, pp. 207-216, 1996.

[5] J. Knapik, "Physiological, biomechanical and medical aspects of soldier load carriage," in Proceedings of NATO RTO Meeting 56: Innovations in Load Carriage System Design and Evaluation, Kingston, Canada, 2000, keynote paper 1.

[6] H. Kinoshita, "Effects of different loads and carrying systems on selected biomechanical parameters describing walking gait," Ergonomics, vol. 28, no. 9, pp. 1347-1362, 1985.

[7] P. E. Martin and R. C. Nelson, "The effect of carried loads on the walking patterns of men and women," Ergonomics, vol. 29, no. 10, pp. 1191-1202, 1986.

[8] E. Harman, K. H. Han, P. Frykman, M. Johnson, F. Russell, and M. Rosenstein, "The effects on gait timing, kinetics and muscle activity of various loads carried on the back," Medicine and Science in Sports and Exercise, vol. 24, p. S129, 1992.

[9] D. C. Tilbury-Davis and R. H. Hooper, "The kinetic and kinematic effects of increasing load carriage upon the lower limb," Human Movement Science, vol. 18, no. 5, pp. 693-700, 1999.

[10] M. LaFiandra, R. C. Wagenaar, K. G. Holt, and J. P. Obusek, "How do load carriage and walking speed influence trunk coordination and stride parameters?" Journal of Biomechanics, vol. 36, no. 1, pp. 87-95, 2003.

[11] G. M. U. Ghori and R. G. Luckwill, "Responses of the lower limb to load carrying in walking man," European Journal of Applied Physiology and Occupational Physiology, vol. 54, no. 2, pp. 145150, 1985.

[12] J. Knapik, K. Reynolds, J. Staab, J. A. Vogel, and B. Jones, "Injuries associated with strenuous road marching," Military Medicine, vol. 157, no. 2, pp. 64-67, 1992.

[13] W. J. Wilson, "Brachial plexus palsy in basic trainees," Military Medicine, vol. 152, no. 10, pp. 519-522, 1987.

[14] M. Holewijn, "Physiological strain due to load carrying," European Journal of Applied Physiology and Occupational Physiology, vol. 61, no. 3-4, pp. 237-245, 1990.

[15] M. Holewijn and T. Meeuwsen, "Physiological strain during load carrying: effects of mass and type of backpack," in Proceedings of NATO RTO Meeting 56: Innovations in Load Carriage System Design and Evaluation, Kingston, Canada, 2000, paper 1 . 
[16] J. Martin and R. Hooper, "Military load carriage: a novel method of interface pressure analysis," in Proceedings of NATO RTO Meeting 56: Innovations in Load Carriage System Design and Evaluation, Kingston, Canada, 2000, paper 22.

[17] R. P. Pelot, A. Rigby, J. M. Stevenson, and J. T. Bryant, "A static biomechanical load carriage model," in Proceedings of NATO RTO Meeting 56: Innovations in Load Carriage System Design and Evaluation, Kingston, Canada, 2000, paper 25.

[18] M. Gretton, Identification of the dynamic suspension characteristics of rucksacks [Ph.D. thesis], The University of Salford, Manchester, UK, 2004.

[19] M. Gretton and D. Howard, "Identifying and modeling the dynamics behaviour of load carriage systems," in Proceedings of NATO RTO Meeting 56: Innovations in Load Carriage System Design and Evaluation, pp. 27-29, Kingston, Canada, 2000.

[20] L. Ren, R. K. Jones, and D. Howard, "Whole body inverse dynamics over a complete gait cycle based only on measured kinematics," Journal of Biomechanics, vol. 41, no. 12, pp. 27502759, 2008.

[21] A. Cappozzo, F. Catani, U. Della Croce, and A. Leardini, "Position and orientation in space of bones during movement: anatomical frame definition and determination," Clinical Biomechanics, vol. 10, no. 4, pp. 171-178, 1995.

[22] P. de Leva, "Adjustments to zatsiorsky-seluyanov's segment inertia parameters," Journal of Biomechanics, vol. 29, no. 9, pp. 1223-1230, 1996.

[23] L. Ren, R. Jones, and D. Howard, "Generalized approach to three-dimensional marker-based motion analysis of biomechanical multi-segment systems," in Proceedings of the International Society of Biomechanics XXth Congress, Cleveland, Ohio, USA, 2005.

[24] J. H. Challis, "A procedure for determining rigid body transformation parameters," Journal of Biomechanics, vol. 28, no. 6, pp. 733-737, 1995. 


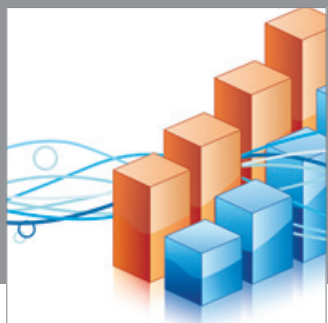

Advances in

Operations Research

mansans

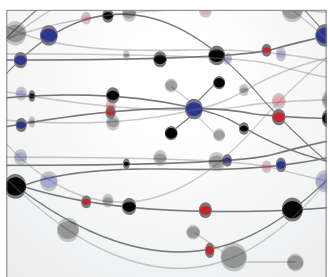

The Scientific World Journal
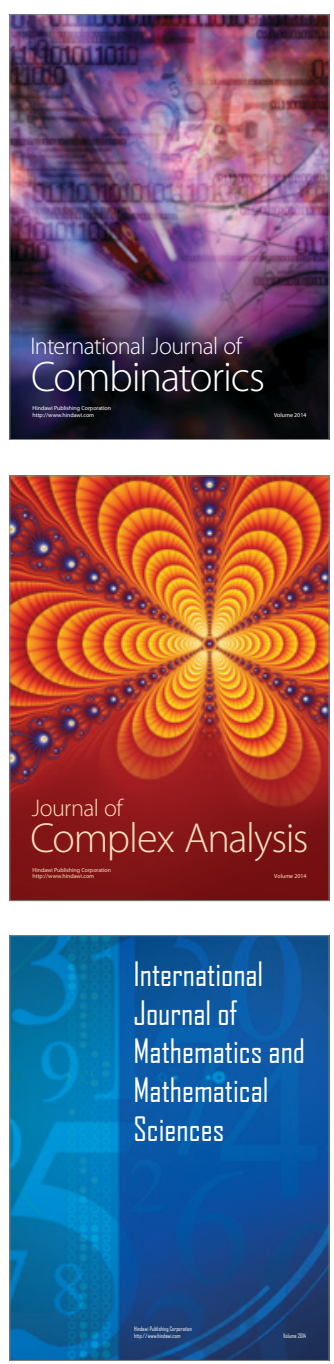
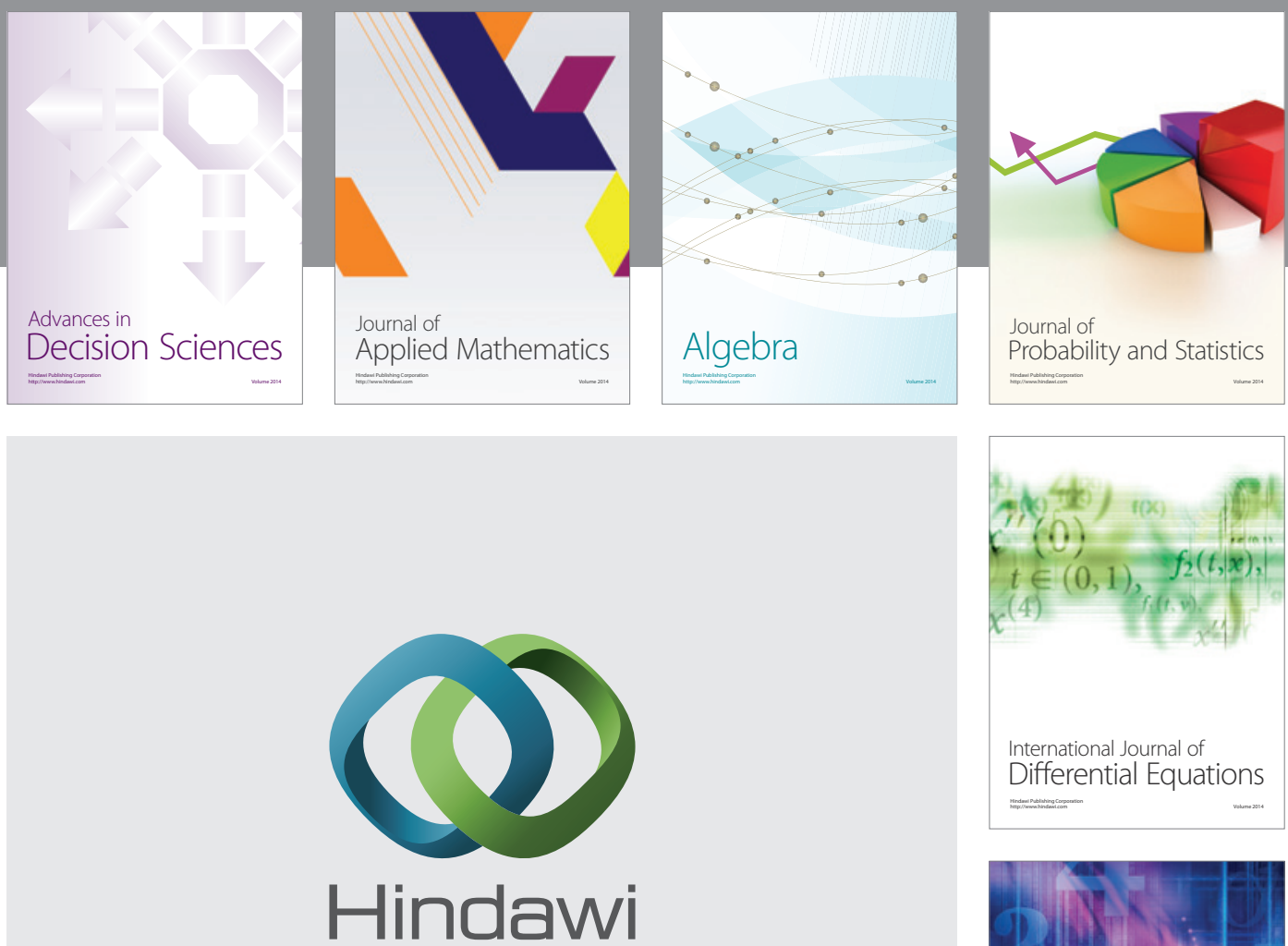

Submit your manuscripts at http://www.hindawi.com
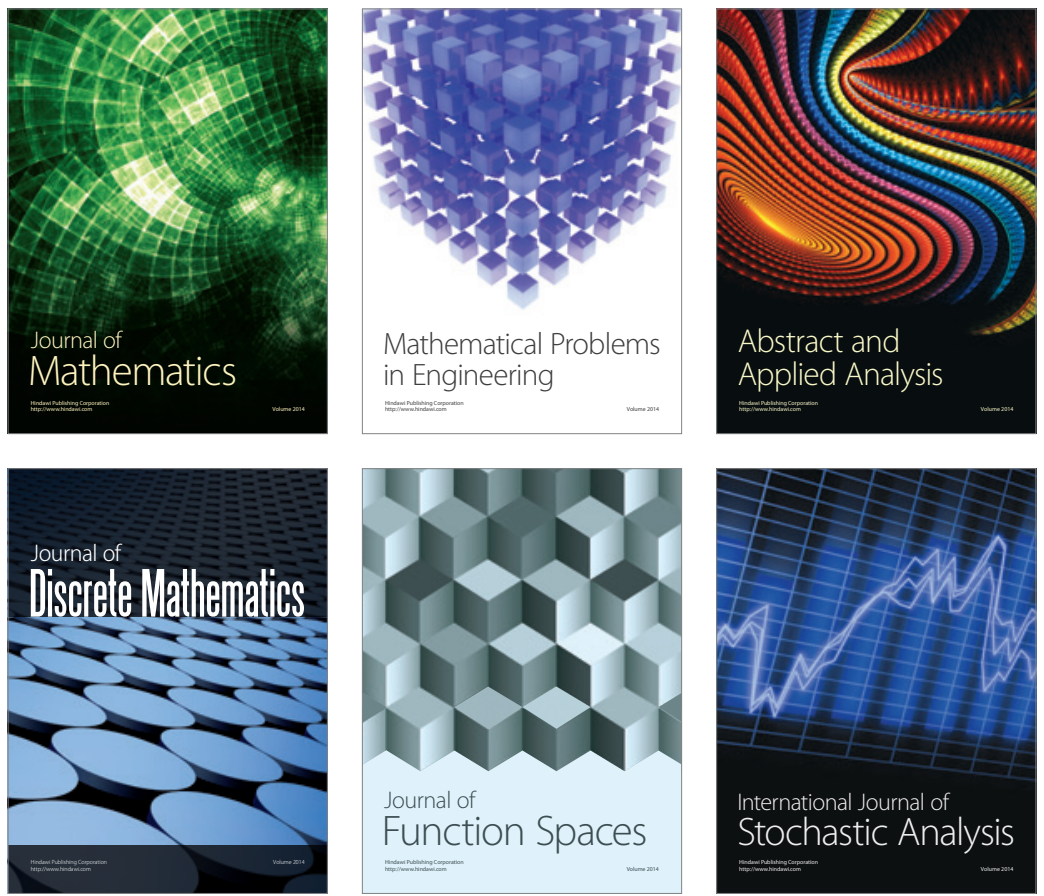

Journal of

Function Spaces

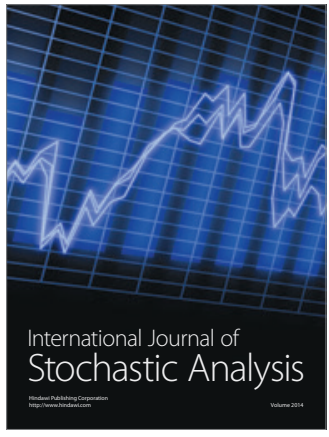

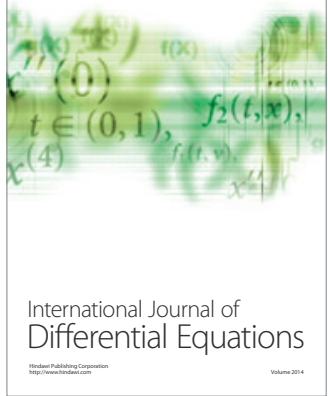
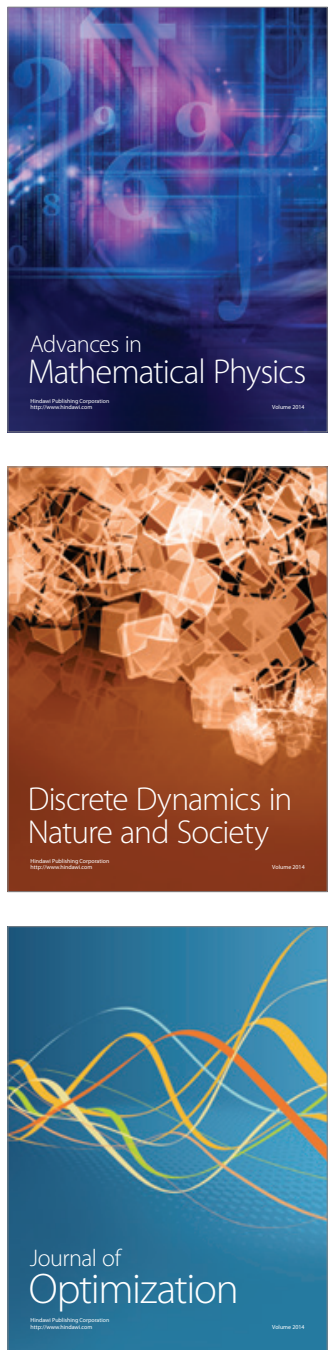